\title{
Biomarkers for Brain Disorders Electrochemically Detected By BRODERICK $\mathrm{PROBE}^{\circledR}$ Microelectrodes/Biosensors
}

\author{
Waqas Saleem ${ }^{1,2}$ and Patricia A Broderick ${ }^{1,2,3 *}$ \\ ${ }^{1}$ Department of Physiology, Pharmacology \& Neuroscience, The Sophie Davis School of Biomedical Education, The City College of New York, The City University of New \\ York, NY, USA \\ ${ }^{2}$ Doctoral Program, Department of Biology, City University of New York Graduate Center, NY, USA \\ ${ }^{3}$ Department of Neurology, NYU Langone School of Medicine, NYU Comprehensive Epilepsy Center, NY, USA

\begin{abstract}
Here, we present results from two independent studies carried out using Neuromolecular Imaging (NMI) with miniature BRODERICK PROBE ${ }^{\circledR}$ biosensors. In the first study, we imaged neurotransmitters and neurochemicals in human epilepsy patients intraoperatively during early and late neurodegeneration. In the second study, we imaged neurotransmitters and neurochemicals in an experimental murine model using animals with and without neurodegeneration caused by Parkinson's disease (PD). We compared our results derived from animals with lesioned group (PD) with non-lesioned group (non-PD), using the same in vivo NMI paradigm. NMI biotechnology enabled neurotransmitters, neuropeptides and neurochemical imaging of dopamine (DA), serotonin (5-HT), homovanillic acid (HVA), L-tryptophan (L-TP), dynorphin A (DYN A) and somatostatin (SRIF). Each neurotransmitter and neurochemical was imaged at its respective signature i.e., its electroactive oxidation/half-wave potential. Results showed neuropeptide signatures of DYN A and SRIF as common biomarker molecules following late neurodegeneration in epilepsy patients and in PD animal models. Placing these two studies together allowed us to us to provide a new hypothesis about a possible biomarker link between the two neurodegenerative diseases, epilepsy and PD. Interestingly, this biomarker link, to our knowledge has not been observed previously. These findings will provide new strategies for better diagnoses, detection of and protection against epilepsy and Parkinson's disease.
\end{abstract}

Keywords: Neurodegeneration; Biomarker; Neuromolecular Imaging; Epilepsy; Neurotransmitter; Parkinson's Disease; Human hippocampus; Dopamine; Serotonin; Dynorphin; Somatostatin; L-tryptophan; Homovanillic acid; Neuropeptides; Brain injury; Microelectrode

\section{Introduction}

Progressive cell death in brain neuronal circuits occurs far too often from brain injuries caused by diseases such as Epilepsy, Parkinson's disease and Alzheimer's disease. Epilepsy is a neurological disorder characterized by repeated seizures over time [1]. In this disorder, the brain exists in an excitable state, increasing neuronal firing rates resulting in abnormally repeated signals accompanied by seizures. Epilepsy may result due to various causes such as stroke, dementia, traumatic brain injury, infections (meningitis, encephalitis, and AIDS), brain tumor, brain injury and congenital brain defect.

Parkinson's disease (PD) is the second most common progressive neurodegenerative disease in America [2]. It is characterized by the formation of Lewy bodies and Lewy neurites in dopaminergic (DA) neurons of substantia nigra pars compacta (SNpc) of brain. Symptoms include progressive impaired movement as (a) uncontrollable resting tremors (b) bradykinesia, (c) muscle rigidity and (d) postural instability. Consistent loss of DA neurons results in the depletion of dopamine level of brainstem and striatal projections, involved in motor function of CNS.

Several animal model studies have been conducted to provide further insight into PD and its therapies. However, more experimental approaches are required to elucidate the precise mechanism of action of PD involved with DA neurons in nigrostriatal pathways. One of the major obstacles in the human studies of PD is that brain materials from early or untreated PD patients are unavailable. In addition, there are minimal amounts of animal studies with few published reports to date about in vivo dynamic release of neurotransmitters, neurochemicals and/or neuropeptides in PD animals. Neuromolecular Imaging (NMI) is the first in vivo, real time imaging technique using BRODERICK PROBE $^{\circledR}$ biosensor to image concentrations of different critical neurotransmitters involved in stroke, epilepsy and Parkinson's disease.

Here, we present studies that are primarily focusing over the real time changes associated with brains following neuro-degeneration in human epilepsy patients (early v/s late neurodegeneration) and PD v/s non-PD animal models. We are the first to demonstrate appearance of neuropeptides as hallmark of neurodegeneration and related biomarkers for epilepsy and PD.

\section{Principle of neuromolecular imaging}

Figure 1 illustrates the NMI setup using BRODERICK PROBE biosensor: These biosensors have been tested in controlled studies and details of NMI biotechnology and circuitry are already published [3-13]. NMI works on utilizing electron transfer kinetics by selecting an image of a specific neurochemical or neurotransmitter molecule at an electroactive oxidation/half wave potential. Specific biosensor properties such as hydrophobicity, hydrophilicity, side chains of amino acids of biosensor formulation determine current derived from electron transfer kinetics upon interaction with specific neurotransmitters and

\footnotetext{
*Corresponding author: Patricia A. Broderick, Department of Physiology Pharmacology \& Neuroscience, The Sophie Davis School of Biomedical Education The City College of New York, 160 Convent Ave., New York-10031, USA, Tel: +1-212-650-5479; Fax:+1-212-650-7305; E-mail: broderick@med.cuny.edu Patricia.Broderick@nyumc.org
}

Received March 05, 2013; Accepted April 22, 2013; Published April 24, 2013

Citation: Saleem W, Broderick PA (2013) Biomarkers for Brain Disorders Electrochemically Detected By BRODERICK PROBE ${ }^{\circledR}$ Microelectrodes/Biosensors. J Biosens Bioelectron S12: 003. doi:10.4172/2155-6210.S12-003

Copyright: (c) 2013 Saleem W, et al. This is an open-access article distributed under the terms of the Creative Commons Attribution License, which permits unrestricted use, distribution, and reproduction in any medium, provided the original author and source are credited. 


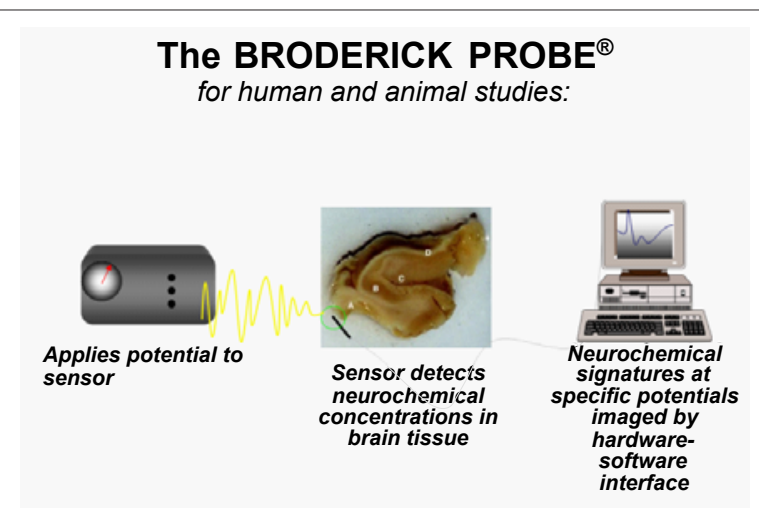

Figure 1: Schematics of NMI setup. BRODERICK PROBE ${ }^{\circledR}$ is implanted to live brain tissue and then externally connected to the potentiostat to apply potential. Upon interaction with specific neurochemical, the BRODERICK $\mathrm{PROBE}^{\circledR}$ transfers its signature electroactive oxidation potential to hardwaresoftware interface for data interpretation and recording.

neuropeptides. The electroactive signature for each molecule is detected by a detector/potentiostat in subunits of volts and amperes, depending upon the type of electronic circuitry chosen in the potentiostat for a particular study. Potentiostat is connected to an external chart recorder to record signals obtained in form of signature oxidation/half wave potential. For both circuitry i.e., linear or semi-differential/semiderivative, $\mathrm{x}$-axis represents voltage in millivolts and $\mathrm{y}$-axis represents current in microamperes.

In vivo studies of brain neurotransmitters based on electrochemistry have been in progress [14-16] and advancements have been made in voltammetry and microvoltammetry using carbon fiber electrodes $[17,18]$. The basic electrochemical principles of voltammetry are listed below:

- To measure the current as a function of applied voltage, when an electroactive species undergo redox reaction at a characteristic redox potential.

- Formulae: $\mathrm{O}+\mathrm{ne}-\leftrightarrow \mathrm{R}$, where, ne=number of electrons, $\mathrm{O}=$ oxidation, $\mathrm{R}=$ reduction.

- Current produced by specific redox reaction is directly proportional to concentration of neurotransmitter, given by the Cottrell equation described below:

$$
i_{t}=n F A C_{o} D_{o}^{1 / 2} / 3.14^{1 / 2} t^{1 / 2}
$$

where:

$\mathrm{o}=$ concentration of electroactive species oxidized

$\mathrm{i}=$ current at time, $\mathrm{t}$

$\mathrm{n}=$ number of electron transfers, eq $/ \mathrm{mol}$

$\mathrm{F}=$ Faraday's constant, $96486 \mathrm{C} / \mathrm{eq}$

$\mathrm{A}=$ electrode area, $\mathrm{cm}^{2}$

$\mathrm{C}=$ concentration of $\mathrm{O}, \mathrm{mol} / \mathrm{cm}^{3}$

$\mathrm{D}=$ Diffusion coefficient of $\mathrm{O}, \mathrm{cm}^{2} / \mathrm{s}$

Conventional voltammetry and microvoltammetry methods since 1970's have validated that the flow of charge (current in amperes) through the surface of indicator is directly proportional to concentration of electroactive species. Relationship in term of charge, current, concentration of electroactive species, time, diffusion layer, size of indicator electrode, electron transfer and Faraday's constant are described below in term of derived equations:

$$
\begin{aligned}
& Q=n F V C_{0 R} \\
& i=d Q / d t \\
& i=n F V d C_{R, t} / d t
\end{aligned}
$$

where:

$\mathrm{V}=$ volume of the diffusion layer on the electrode of measurement $\mathrm{n}=$ number of transferred electrons

$\mathrm{F}=$ Faraday's Constant

$\mathrm{C}_{\mathrm{o}}=$ initial concentration

\section{Material and Methods}

\section{NMI, intra-operative clinical studies in epilepsy patients}

NMI, based on electrochemical principles, has made major advances in the field of nanobiotechnology, science and medicine. For example, one of these cutting-edge advancements made by NMI and the BRODERICK $P R O B E^{\circledR}$ is in the field of human epilepsy intraoperatively in NYU Tisch Hospital with Institutional Review Board (IRB) approval (Figure 2).

Studies in real time with NMI and BRODERICK PROBE biosensors begin with electroencephalographic (EEG) monitoring wherein the site of cortical resection is defined with subdural grid epilepsy electrodes which are placed on the surface of the brain and epilepsy strip electrodes are placed subtemporally.

Then, BRODERICK PROBE ${ }^{\circledR}$ biosensors, with a diameter over five times smaller than epilepsy depth electrodes for invasive EEG, are placed by direct visualization in the exposed cortical region with and without epileptic spike activity in regions destined for resection.

About six to ten recordings with $\gamma$-irradiated [11.6-12.7 kilograys (kGys)], laminar biocompatible carbon-based BRODERICK PROBE ${ }^{\circledR}$

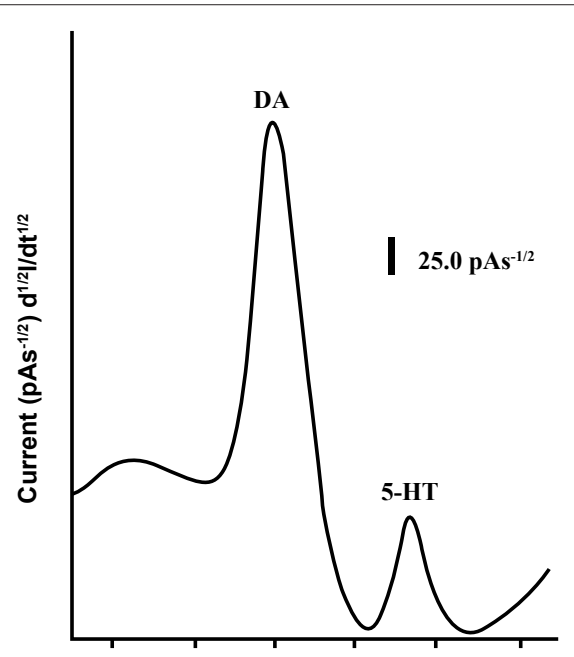

Figure 2: NMI results from resected epileptogenic tissue of hippocampal pyramidal layer (Gray matter) in early neurodegeneration: Monoamines: Dopamine (DA), Serotonin (5-HT). On the x-axis: oxidation potential in term of applied voltage in volts $(\mathrm{V})$. On the $y$-axis: current in picoamperes (pA). Note that the $\mathrm{x}$-axis terminates at approximately $0.45 \mathrm{~V}$ due to the low detection limit of L-tryptophan and neuropeptides in early neurodegeneration. 
biosensors are taken at a cortical micron depth for about 20-30 min time period.

\section{NMI studies in PD and non-PD animal model}

A classical animal model of PD was used to study endogenous brain mechanisms of PD in Sprague-Dawley rat (Rattus norvegicus). Depletion of DA was produced by injecting the neurotoxin, 6-hydoxydopamine (6-OHDA) into substantia nigra (SN) of male, Sprague-Dawley Rattus norvegicus in Charles River Laboratories, North Carolina, USA. This leads to the death of lesioned DA neurons and subsequently to disruption in dorsal striatum (DStr) and hence depletion of DA in DStr. The lesion was made in the substantia nigra (SN) of rats under anesthesia. Stereotaxic coordinates were $\mathrm{AP}=-1.5, \mathrm{ML}=+1.8, \mathrm{DV}=7.5$ [19].

Another group of animals, same weight (approx. 300 grams) and age, did not undergo surgery for lesions in SN. These were the controls or non lesioned groups (Non-PD).

For NMI studies, BRODERICK PROBE ${ }^{\circledR}$ biosensors were implanted in both PD (lesioned) and non-PD (non-lesioned) animals in DStr under sodium pentobarbital anesthesia (50 mg/kg ip) [20]. Protocols for NMI studies with PD and non-PD were performed at Dr. Broderick's laboratory with approval from the Institutional Animal Care and Use Committee (IACUC). These studies were performed to compare endogenous neurochemical profiles of $\mathrm{PD}$ and non-PD animal model.

\section{Results and Discussion}

\section{Clinical studies with epilepsy patient, in situ and in vivo}

During early neurodegeneration, monoamines are predominant in resected epileptogenic tissue of brain (Gray matter) as evident from NMI results from the BRODERICK $\mathrm{PROBE}^{\circledR}$ biosensor. Results showed a relatively high concentration of DA compared to 5-HT were present in patient's hippocampal pyramidal layer of brain. Lauric acid is a saturated fatty acid with a 12-carbon atom chain. It has a hydrophobic head with hydrophilic tail which helps to act as a surfactant to reduce surface tension. This surfactant also acts to assist the migration of molecules to form an oriented, adsorbed film on the interfacial surface of the indicator sensor.

Figure 3 depicts recordings from actual human epilepsy neocortex during intraoperative surgery. Epileptogenic tissue recorded from

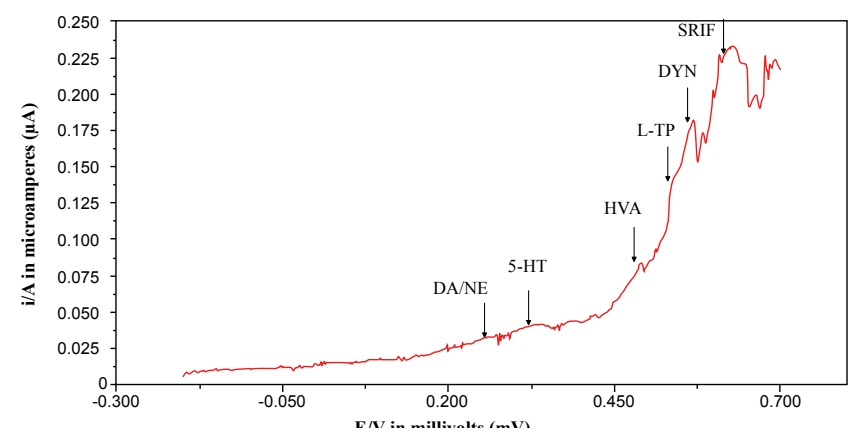

Figure 3: In vivo, NMI results from actual epilepsy patient studied intraoperatively showing late neurodegeneration. On the x-axis: oxidation potential $(E / V)$ in millivolts $(m V)$. On the $y$-axis: current (i/A) in microamperes. Minimal concentrations of dopamine (DA), homovanillic acid (HVA), serotonin (5-HT), and high concentrations of L-tryptophan (L-TP) (precursor to 5-HT), and neuropeptides (dynorphin and somatostatin) are present.

\begin{tabular}{|l|l|c|c|c|}
\hline \multicolumn{2}{|c|}{} & $\begin{array}{c}\text { Detection } \\
\text { Limits-In vitro }\end{array}$ & $\begin{array}{c}\text { Detection } \\
\text { Limits-In vivo }\end{array}$ & $\begin{array}{c}\text { Approximate } \\
\text { Neurochemical } \\
\text { Concentration }\end{array}$ \\
\hline Catecholamine & DA & $2 \mathrm{nM}$ & $20 \mathrm{nM}$ & $\sim 0.2 \mathrm{nM}$ \\
\hline Serotonin & $5-\mathrm{HT}$ & $1 \mathrm{nM}$ & $10 \mathrm{nM}$ & $\sim 0.5 \mathrm{nM}$ \\
\hline Homovanillic Acid & HVA & $20 \mathrm{nM}$ & $0.2 \mu \mathrm{M}$ & $7 \mathrm{nM}$ \\
\hline Tryptophan & L-TP & $20 \mathrm{nM}$ & $0.2 \mu \mathrm{M}$ & $1 \mu \mathrm{M}$ \\
\hline Dynorphin & DYN & $4 \mathrm{nM}$ & $40 \mathrm{nM}$ & $2 \mu \mathrm{M}$ \\
\hline Somatostatin & SRIF & $3.7 \mathrm{nM}$ & $37 \mathrm{nM}$ & $4 \mu \mathrm{M}$ \\
\hline
\end{tabular}

Table 1: Preliminary data: detection limits for compounds studied intraoperatively.

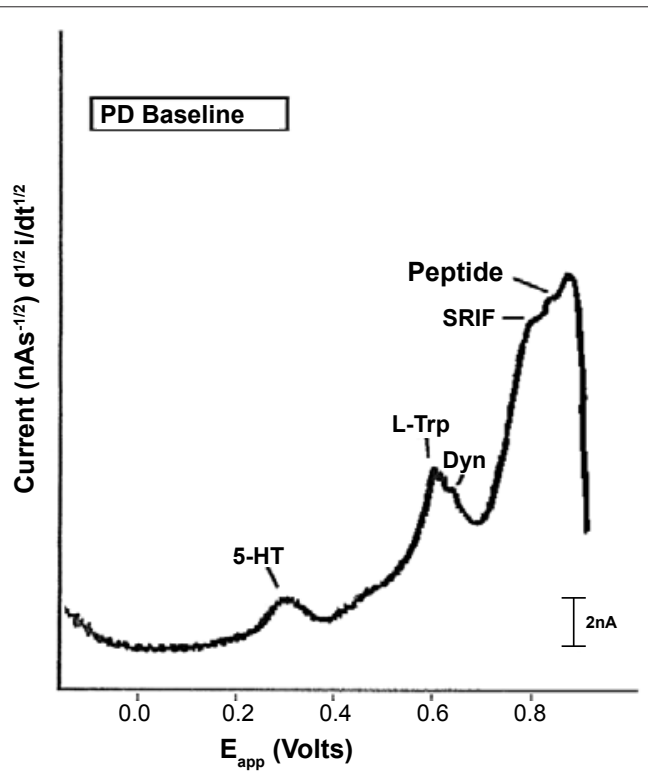

Figure 4: NMI results from lesioned group (PD animal model) with endogenous neurochemical profile. On the x-axis: oxidation potential in term of applied voltage in volts $(V)$. On the $y$-axis: current in nanoamperes (nA). NMI was conducted using the BRODERICK PROBE ${ }^{\circledR}$ laurate biosensor. Serotonin (5$\mathrm{HT}$ ), L-tryptophan (L-Trp) and neuropeptides (dynorphin and somatostatin) were present. Note that dopamine (DA) neurotransmitter was absent in lesioned group (PD).

brain shows late neurodegeneration. A predominance of L-tryptophan (L-TP) and neuropeptides DYN A and SRIF over monoamines are observed. Therefore, results show that L-TP and neuropeptides (DYN A and SRIF) may be biomarkers for neurodegeneration.

Intraoperative images were verified by comparison with a fourteen year empirical database on epilepsy. Table 1 represents preliminary data of detection limits for different compounds that were studied intraoperatively using the NMI biotechnology.

Preclinical studies in animal models (PD v/s. non-PD, in situ, in vivo)

Figure 4 depicts an abundant presence of neurotransmitter peptides, neuropeptides and a lower concentration of the indoleamine, 5-HT, while DA, expectedly, was not imaged in any PD animal model. Meanwhile, the non-lesioned group in figure 5 exhibited significant concentrations of monoamines, DA and 5-HT while neuropeptides were not observed in any non-PD animal model.

\section{Conclusion}

Results showed that the precursor to 5-HT, which is L-TP, and the neuropeptides DYN A and SRIF may be biomarkers for 


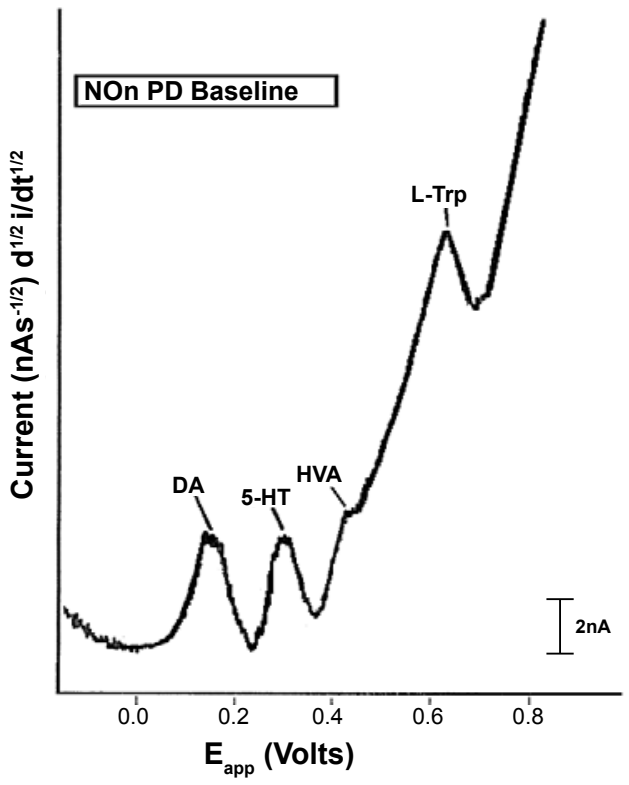

Figure 5: NMI results from controls, the non-lesioned group (Non-PD animal model) with endogenous neurochemical profile. On the x-axis: oxidation potential in term of applied voltage in volts (V). On the $y$-axis: current in nanoamperes $(\mathrm{nA})$. NMI was conducted using the BRODERICK PROBE laurate biosensor. Dopamine (DA), serotonin (5-HT) and L-tryptophan (L-Trp) were present in high concentration with minimal homovanillic acid (HVA). Neuropeptides were completely absent in non lesioned group (non-PD).

neurodegeneration. The appearance of L-TP and neuropeptides in late neurodegeneration of epilepsy patients and in the lesioned group (PD animal model) support our proposal that neuropeptides appear as biomarkers of neurodegeneration. We are the first to propose peptide appearance as biomarker molecule following neurodegeneration in epilepsy patients and Parkinson's disease. Furthermore, since DYN A is comprised of glutamate and SRIF has been shown to alleviate the symptoms of PD in animal models [20,21], we simply suggest that DYN A may be the excitatory molecule in neurodegeneration whereas SRIF may provide neuroprotection in PD. Our findings provide new avenues for effective diagnoses and detection by using these common biomarkers against neurodegenerative disorders.

\section{Acknowledgements}

This work represents a contribution by the senior author, $\mathrm{Dr}$. Patricia A Broderick, as invited speaker at The International Congress and Exhibition on Biosensors \& Bioelectronics May 13-17 $7^{\text {th }}, 2012$, Las Vegas, Nevada, USA. The Conference was sponsored by OMICS group. This work was also presented in poster form at the Institute of Electrical and Electronics Engineers (IEEE) Conference at the City College of New York (CCNY) sponsored by the Department of Biomedical Engineering at the Grove School of Engineering, CCNY, in concert with the NYU Polytechnic Institute.

Waqas Saleem, B.S. is a candidate for the Master's Degree in Biology, CCNY, CUNY. Waqas is performing research in Dr. Broderick's laboratory in the Department of Physiology, Pharmacology and Neuroscience at the Sophie Davis School of Biomedical Education, CCNY, CUNY.

The authors wish to thank the Broderick Brain Foundation, the F.M. Kirby Foundation, Foundation for Children with Epileptic Seizures (FACES), The Leon Lowenstein Foundation and the MacKenzie Foundation for funding support for our laboratory during these studies.

\section{References}

1. Chang BS, Lowenstein DH (2003) Epilepsy. N Engl J Med 349: 1257-1266.

2. Mathias CJ (2008) L-dihydroxyphenylserine (Droxidopa) in the treatment of orthostatic hypotension: the European experience. Clin Auton Res 18: 25-29.
3. Broderick P, Ho H, Wat K, Murthy V (2008) Laurate Biosensors Image Brain Neurotransmitters In Vivo: Can an Antihypertensive Medication Alter Psychostimulant Behavior? Sensors 8: 4033-4061.

4. Broderick PA, Pacia SV (2005) Imaging White Matter Signals in Epilepsy Patients. Bioimaging in Neurodegeneration 199-206.

5. Broderick PA, Pacia SV (2006) Identification, diagnosis, and treatment of neuropathologies, neurotoxicities, tumors and brain and spinal cord injuries using electrodes with microvoltammetry. US Patent Application 20120029331.

6. Broderick PA, Pacia SV (2007) Identification, diagnosis, and treatment of neuropathologies, neurotoxicities, tumors and brain and spinal cord injuries using microelectrodes with microvoltammetry. US Patent No: 7,112,319.

7. Broderick PA (1988) Distinguishing in vitro electrochemical signatures for norepinephrine and dopamine. Neurosci Lett 95: 275-280.

8. Broderick PA (1989) Characterizing stearate probes in vitro for the electrochemical detection of dopamine and serotonin. Brain Res 495: 115-121.

9. Broderick PA (1989) Cathodic Electrochemical Current Arrangement with Telemetric Application. US4883057 A.

10. Broderick PA (1995) Microelectrodes and their use in cathodic electrochemical current arrangement with telemetric application. US Patent \# 5,433,710.

11. Broderick PA (1999) Microelectrodes and their use in an electrochemical arrangement with telemetric application. US Patent \# 5, 938, 903.

12. Broderick PA (2008) Studies of oxidative stress mechanisms using a morphine/ ascorbate animal model and novel $\mathrm{N}$-stearoyl cerebroside and laurate sensors. J Neural Transm 115: 7-17.

13. Broderick PA, Pacia SV, Doyle WK, Devinsky O (2000) Monoamine neurotransmitters in resected hippocampal subparcellations from neocortical and mesial temporal lobe epilepsy patients: in situ microvoltammetric studies. Brain Res 878: 48-63.

14. Hubbard AT, Anson FC (1970) Electroanalytical Chemistry. (1stedn), Marce Dekker, New York.

15. Adams RN, Marsden CA (1996) New techniques in psychopharmacology. In: Handbook of Psychopharmacology, Iversen, LL, Snyder SH, (eds.). Plenum Press, New York.

16. Kissinger PT, Heineman WR (1996) Laboratory Techniques in Electroanalytical Chemistry. (2ndedn), Marcel Dekker, Inc.

17. David DJ, Zahniser NR, Hoffer BJ, Gerhardt GA (1998) In vivo electrochemical studies of dopamine clearance in subregions of rat nucleus accumbens: differential properties of the core and shell. Exp Neurol 153: 277-286.

18. Garris PA, Christensen JR, Rebec GV, Wightman RM (1997) Real-time measurement of electrically evoked extracellular dopamine in the striatum of freely moving rats. J Neurochem 68: 152-161.

19. Paxinos G, Watson C (2006) The Rat Brain in Stereotaxic Coordinates. (6thedn), Academic Press.

20. Broderick PA, Kolodny EH (2009) Real Time Imaging of Biomarkers in the Parkinson's Brain Using Mini-Implantable Biosensors. II. Pharmaceutical Therapy with Bromocriptine. Pharmaceuticals 2: 236-249.

21. Lu JQ, StoessI AJ (2002) Somatostatin modulates the behavioral effects of dopamine receptor activation in parkinsonian rats. Neuroscience 112: 261-266. 